\title{
Self-Force on a Particle in Orbit around a Black Hole
}

\author{
Lior M. Burko \\ Theoretical Astrophysics, California Institute of Technology, Pasadena, California 91125
}

(Received 11 November 1999)

\begin{abstract}
We study the self-force acting on a scalar charge in uniform circular motion around a Schwarzschild black hole. The analysis is based on a direct calculation of the self-force via mode decomposition, and on a regularization procedure based on Ori's mode-sum regularization prescription. We find the four self-forces at arbitrary radii and angular velocities (both geodesic and nongeodesic), in particular near the black hole, where general-relativistic effects are strongest, and for fast motion. We find the radial component of the self-force to be repulsive or attractive, depending on the orbit.
\end{abstract}

PACS numbers: 04.25.-g, 04.30.Db, 04.70.Bw

The problem of finding the equations of motion for a particle in curved spacetime has recently become extremely important, as the first generation of interferometric gravitational wave detectors will soon be operational, and with the prospects of having a gravitational wave space antenna in the not-very-distant future. The generation of very accurate templates for the waveforms detected from a system of a compact object in orbit around a supermassive black hole is an extremely hard task. It is likely that one would need to have accurate templates for as many as $5 \times 10^{5}$ orbits. For such a system, accurate templates are necessary for detection, because the predicted signal-to-noise ratio for LISA is approximately of order 10 for a 1 year integration time. Lack of accurate templates would result in a loss of a factor of roughly the square root of the number of orbits in sensitivity [1], which would result in signal-to-noise ratio below the detectibility threshold.

In order to generate accurate templates, an important necessary ingredient is the inclusion of radiation reaction in the orbital evolution of the compact object. The radiation-reaction forces need to be calculated locally, i.e., in the neighborhood of the compact object. In the conventional approach, one calculates, at infinity and at the event horizon of the black hole, the fluxes of quantities, which are constants of motion in the absence of radiation reaction. Then, one uses a balance argument to relate these fluxes to the rate of change of a corresponding local quantity of the compact object. This approach generally fails because of the inadditivity of the Carter constant. For very simple cases, e.g., for circular or equatorial orbits around a Kerr black hole, the evolution of the Carter constant is trivial, such that the conventional approach is useful [2]. However, generic orbits around a rotating black hole are neither circular nor equatorial, and consequently a new approach, which is not based on balance arguments, is of great need.

Several approaches have been suggested for the calculation of self-forces. Quinn and Wald [3] and Mino, Sasaki, and Tanaka [4] recently proposed general approaches for the calculation of self-forces. However, it is not presently clear how to apply these approaches directly for actual computations, the greatest problem being the calculation of the so-called "tail" term of the Green's function, which arises from the failure of the Huygens principle in curved spacetime. (In addition, there are in general also local, Ricci-curvature coupled, and Abraham-Lorentz-Dirac (ALD) type terms [5], which are much easier to calculate, the former vanishing identically in vacuum.)

Recently, Ori proposed a local, causal approach for the calculation of the self-forces [6-8], which is based on decomposition of the self-force into modes, and on a mode-sum regularization prescription (MSRP). Although MSRP is not fully proven as yet, it has already been shown to be valid for simple cases, such as scalar charges in general orbits in Schwarzschild spacetime, and, in particular, for circular orbits which we consider here. MSRP is likely to be susceptible to generalization also for massive particles in orbit around a Kerr black hole. If robust, MSRP can be of great importance for the generation of accurate templates. We hope that MSRP can be combined with other approaches, which were recently proposed, such as mode decomposition of the self-forces which are sourced by just the distant past world line [9] or a normalneighborhood expansion [10].

We first describe very briefly the main ideas of MSRP [7,8]. Then, we apply MSRP for the case of a scalar particle in circular orbit around a Schwarzschild black hole, and calculate the self-four-force acting on the particle linearized in its own self-field.

The contribution to the physical self-force from the tail part of the Green's function can be decomposed into stationary Teukolsky modes, and then summed over the frequencies $\omega$ and the azimuthal numbers $m$. The self-force equals then the limit $\epsilon \rightarrow 0^{-}$of the sum over all $\ell$ modes, of the difference between the force sourced by the entire world line (the bare force bare $F_{\mu}^{\ell}$ ) and the force sourced by the half-infinite world line to the future of $\epsilon$, where the particle has proper time $\tau=0$, and $\epsilon$ is an event along the past $(\tau<0)$ world line. Next, we seek a regularization function $h_{\mu}^{\ell}$ which is independent of $\epsilon$, such that the series $\sum_{\ell}\left({ }^{\text {bare }} F_{\mu}^{\ell}-h_{\mu}^{\ell}\right)$ converges. Once such a function is found, the regularized self-force is then given by ${ }^{\text {ren }} F_{\mu}=\sum_{\ell}\left({ }^{\text {bare }} F_{\mu}^{\ell}-h_{\mu}^{\ell}\right)-d_{\mu}$, where $d_{\mu}$ is a finite valued function. MSRP $[7,8]$ then shows, from a local 
integration of the Green's function, that the regularization function $h_{\mu}^{\ell}=a_{\mu} \ell+b_{\mu}+c_{\mu} \ell^{-1}$, and for the case of a scalar charge in circular orbit around a Schwarzschild black hole MSRP yields the values of the functions $a_{\mu}$, $b_{\mu}, c_{\mu}$, and $d_{\mu}$ analytically. In particular, it can be shown that for such orbits $a_{\mu}=0=c_{\mu}$ and $d_{r}=0$, such that in practice the regularization prescription of the radial force is reduced to subtracting $b_{r}$ from each $\ell$ mode of the bare radial force. Note that $b_{\mu}$ is just the limit $\ell \rightarrow \infty$ of bare $F_{\mu}^{\ell}$.

In the following we describe the results obtained from this new approach for the case of a pointlike scalar charge in circular orbit around a Schwarzschild black hole. Our results are fully relativistic, i.e., we do not introduce any simplifying assumptions such as far field or slow motion. Because of the fully relativistic nature of this study, our analysis is numerical. However, it is reasonable to expect that analytical solutions will not be available in general, except, possibly, only for very simple cases, such as static configurations [11]. We calculate the contribution to the force which the scalar charge feels, due to its own field, to leading order in the particle's charge. We use spherical Regge-Wheeler coordinates, for which the Schwarzschild metric is $d s^{2}=\left(1-\frac{2 M}{r}\right)\left(-d t^{2}+d r^{* 2}\right)+r^{2}\left(d \theta^{2}+\right.$ $\sin ^{2} \theta d \phi^{2}$ ), where $M$ is the black hole's mass, and the radial Schwarzschild coordinate $r\left(r^{*}\right)$ is given implicitly by $r^{*}=r+2 M \ln |r /(2 M)-1|$. The field satisfies the wave equation $\nabla_{\mu} \nabla^{\mu} \Phi\left(x^{\alpha}\right)=-4 \pi \rho\left(x^{\alpha}\right)$, where the charge density $\rho\left(x^{\alpha}\right)=q \int_{-\infty}^{\infty} d \tau \delta^{4}\left[x^{\alpha}-\right.$ $\left.x_{0}^{\alpha}(\tau)\right](-g)^{-1 / 2}$. Here, $q$ is the charge of the particle, whose world line is $x_{0}^{\alpha}(\tau), \tau$ being its proper time, $g$ being the metric determinant, and $\nabla_{\mu}$ denotes covariant differentiation. We take the charge to be in circular orbit at $r^{*}=r_{0}^{*}, \theta=\pi / 2$, and $d \phi / d t=\Omega$. (We are not restricted to the Keplerian angular velocity $\Omega_{K}$.) We next decompose the scalar field $\Phi$ into modes according to $\Phi=\int_{-\infty}^{\infty} d \omega \sum_{\ell m} e^{-i \omega t} \Psi_{\ell m}\left(r^{*}\right) Y^{\ell m}(\theta, \phi) / r\left(r^{*}\right)$, such that the equation which governs the field $\Psi_{\ell m}$ becomes

$$
\begin{aligned}
& \frac{d^{2} \Psi_{\ell m}}{d r^{* 2}}+\left\{\omega^{2}-V_{\ell}\left[r\left(r^{*}\right)\right]\right\} \Psi_{\ell m} \\
=- & -4 \pi \frac{q}{\gamma} \frac{\delta\left(r-r_{0}\right)}{r_{0}} \delta(\omega-m \Omega) Y^{\ell m}(\pi / 2, \phi) e^{-i m \phi} .
\end{aligned}
$$

This equation should be solved for each mode $\ell m$ with boundary conditions of ingoing waves at the event horizon $\left(r^{*} \rightarrow-\infty\right)$, and outgoing waves at infinity $\left(r^{*} \rightarrow \infty\right)$. The effective potential is given by $V_{\ell}(r)=(1-2 M / r)\left[2 M / r^{3}+\ell(\ell+1) / r^{2}\right], \quad$ and $\gamma=1 / \sqrt{1-2 M / r-r^{2} \Omega^{2}}$. The contribution of the $\ell m$ mode to the force is given by bare $F_{\mu}^{\ell m}=$ $q\left(\Phi_{, \mu}^{\ell m}+u_{\mu} u^{\nu} \Phi_{, \nu}^{\ell m}\right)$ (note that for circular orbits $\left.u^{\nu} \Phi_{, \nu}^{\ell m}=0\right)$.

We next find numerically the solutions $\Psi_{\ell m}^{+}\left(\Psi_{\ell m}^{-}\right)$for the homogeneous equations corresponding to Eq. (1), which satisfy the boundary condition at infinity (the horizon). The components of the force are then given by

$$
\begin{aligned}
{ }^{\text {bare }} F_{r^{*}}^{\ell m}=2 \pi q^{2} \frac{\left|Y^{\ell m}\left(\frac{\pi}{2}, 0\right)\right|^{2}}{\gamma r_{0}^{2}}\{ & -\operatorname{Re}\left[W_{\ell m}^{-1}\left(r_{0}^{*}\right)\right] \operatorname{Re}\left[S_{\ell m}\left(r_{0}^{*}\right)\right]+\frac{2}{r_{0}}\left(1-\frac{2 M}{r_{0}}\right) \operatorname{Re}\left[W_{\ell m}^{-1}\left(r_{0}^{*}\right)\right] \operatorname{Re}\left[T_{\ell m}\left(r_{0}^{*}\right)\right] \\
& \left.-\frac{2}{r_{0}}\left(1-\frac{2 M}{r_{0}}\right) \operatorname{Im}\left[W_{\ell m}^{-1}\left(r_{0}^{*}\right)\right] \operatorname{Im}\left[T_{\ell m}\left(r_{0}^{*}\right)\right]+\operatorname{Im}\left[W_{\ell m}^{-1}\left(r_{0}^{*}\right)\right] \operatorname{Im}\left[S_{\ell m}\left(r_{0}^{*}\right)\right]\right\},
\end{aligned}
$$

where $W_{\ell m}$ is the Wronskian determinant of $\Psi_{\ell m}^{-}\left(r^{*}\right)$ and $\Psi_{\ell m}^{+}\left(r^{*}\right), T_{\ell m}\left(r^{*}\right)=\Psi_{\ell m}^{+}\left(r^{*}\right) \Psi_{\ell m}^{-}\left(r^{*}\right)$, and $S_{\ell m}\left(r^{*}\right)=$ $\Psi_{\ell m}^{+}\left(r^{*}\right) \Psi_{\ell m, r^{*}}^{-}\left(r^{*}\right)+\Psi_{\ell m}^{-}\left(r^{*}\right) \Psi_{\ell m, r^{*}}^{+}\left(r^{*}\right)$. We find that

$$
\begin{aligned}
{ }^{\text {bare }} F_{t}^{\ell m}= & -4 \pi q^{2} m \Omega \frac{\left|Y^{\ell m}\left(\frac{\pi}{2}, 0\right)\right|^{2}}{\gamma r_{0}^{2}} \\
& \times\left\{\operatorname{Im}\left[W_{\ell m}^{-1}\left(r_{0}^{*}\right)\right] \operatorname{Re}\left[T_{\ell m}\left(r_{0}^{*}\right)\right]\right. \\
& \left.+\operatorname{Re}\left[W_{\ell m}^{-1}\left(r_{0}^{*}\right)\right] \operatorname{Im}\left[T_{\ell m}\left(r_{0}^{*}\right)\right]\right\} .
\end{aligned}
$$

We also obtain bare $F_{\phi}^{\ell m}=-\Omega^{-1 \text { bare }} F_{t}^{\ell m}$ and ${ }^{\text {bare }} F_{\theta}^{\ell m}=$ 0 . It is convenient to define new radial functions $Z^{ \pm \ell m}\left(r^{*}\right)$ by $\Psi^{ \pm \ell m}\left(r^{*}\right)=e^{ \pm i \omega r^{*}} Z^{ \pm \ell m}\left(r^{*}\right)$, which satisfy the homogeneous equations

$$
\frac{d^{2} Z^{ \pm \ell m}}{d r^{* 2}} \mp 2 i \omega \frac{d Z^{ \pm \ell m}}{d r^{*}}-V_{\ell}\left[r^{*}(r)\right] Z^{ \pm \ell m}=0,
$$

with boundary conditions $Z^{+\ell m}\left(r^{*} \gg M\right)=$ $1+a_{1}^{+} f_{+}+a_{2}^{+} f_{+}^{2}+O\left(f_{+}^{3}\right)$ and $Z^{-\ell m}\left(r^{*} \ll-M\right)=$ $1+a_{1}^{-} f_{-}+a_{2}^{-} f_{-}^{2}+O\left(f_{-}^{3}\right)$, where $f_{+}=(\omega r)^{-1}$, $f_{-}=1-2 M / r, \quad$ and $\quad a_{1}^{+}=i \ell(\ell+1) / 2, \quad a_{2}^{+}=$ $[-\ell(\ell-1)(\ell+1)(\ell+2)+4 i \omega M] / 8, \quad a_{1}^{-}=[1+$ $\ell(\ell+1)] /(1-4 i \omega M)$, and $a_{2}^{-}=\left[\ell(\ell+1)\left(\ell^{2}+\ell+\right.\right.$ $6)+4 i \omega M+4] /[4(1-2 i \omega M)(1-4 i \omega M)]$. We solve Eq. (4) numerically using both Burlisch-Stoer and fourth-order Runge-Kutta integrations with adaptive step-size controls. Both integrators yield results compatible within the error limits. We place the exterior and interior boundaries at a distance of several mode wavelengths (in $r^{*}$ ) from $r_{0}^{*}$, and then use successive Richardson extrapolations, with increasing distance to the boundaries, until the extrapolation of the boundaries to $r^{*} \rightarrow$ $\pm \infty$ yields an error smaller than a given threshold. Notice that for modes with $m=0$ the wavelength is infinite, such that the boundaries cannot be taken far enough from the charge. Indeed, we find that for this case the Richardson extrapolations do not converge. Instead, we can solve for this case analytically. We find that $f_{t}^{\ell, m=0}=$ $0 \quad$ and $\quad f_{r}^{\ell, m=0}=(2 \pi / \gamma)(q / M)^{2} Y^{\ell 0^{2}}(\pi / 2,0) \times$ $\left[2 Q_{\ell}\left(\rho_{0}\right) d P_{\ell}\left(\rho_{0}\right) / d \rho+1 /\left(1-\rho_{0}^{2}\right)\right], \quad$ where $\rho=$ $(r-M) / M$. Here, $P_{\ell}, Q_{\ell}$ are the Legendre functions of the first and second kinds, respectively. Figure 1 shows the functions $Z_{\ell=1, m=1}^{ \pm}\left(r^{*}\right)$. Similar qualitative behavior 

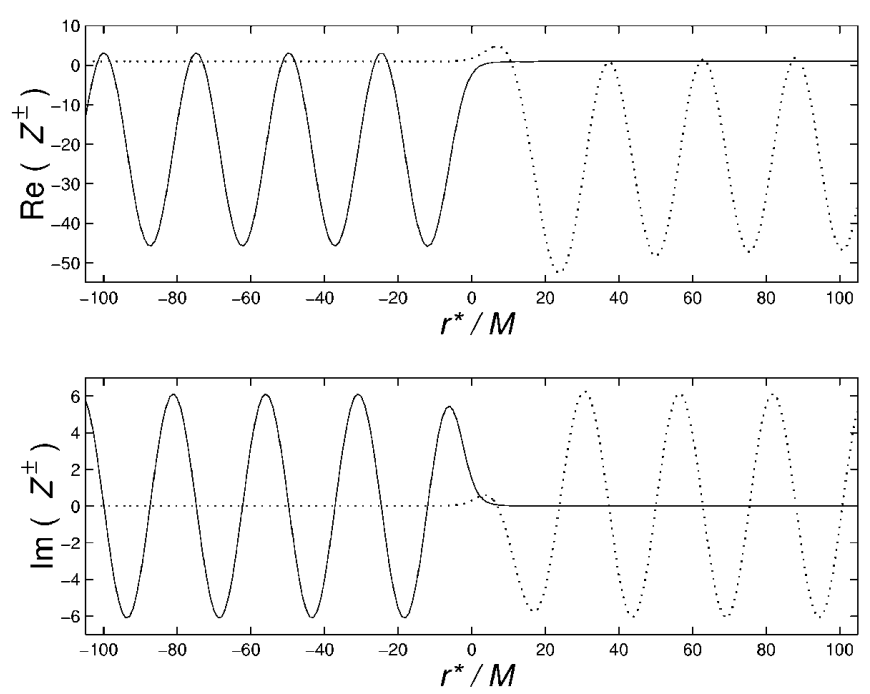

FIG. 1. The radial functions $Z^{ \pm}$for $\ell=1$ and $m=1$ as functions of $r^{*} / M$. The particle is at $r_{0}^{*}=4 M$ and $\Omega=\Omega_{K}$. Top panel: $\operatorname{Re}\left[Z^{+}\left(r^{*}\right)\right]$ (solid line) and $\operatorname{Re}\left[Z^{-}\left(r_{*}\right)\right]$ (dotted line). Bottom panel: Same as in the top panel, for $\operatorname{Im}\left[Z^{ \pm}\left(r_{*}\right)\right]$.

is found also for the other modes. Until the peak of the effective potential barrier the functions $Z^{ \pm \ell m}$ vary only slowly, and then start oscillating rapidly.

The temporal component of the bare force is finite. (In the regularization scheme this corresponds to $b_{t}=$ 0.) MSRP predicts that $d_{t}$ exactly balances the ALD force, such that the full self-force is given only by $F_{t}=$ $\sum_{\ell}{ }^{\text {bare }} F_{t}^{\ell}$. We compare our results with their Minkowski spacetime counterparts. In flat spacetime, one can solve analytically for each mode, sum over all modes, and find that $F_{t}^{\mathrm{Min}}=\frac{1}{3} q^{2} \Omega^{2} r_{0}^{2} \gamma_{\text {Min }}^{5}$. Here, $\gamma_{\text {Min }}$ is the usual flat spacetime Lorentz factor. For the comparison we choose the same values of $r_{0}, \Omega$ for the curved and flat spacetimes. Figure 2 displays $F_{t}$ as a function of $r / M$ for two cases: [Fig. 2(A)] Nongeodesic circular orbits, with a fixed angular velocity $\Omega$. (In this case the tangential velocity increases linearly with $r$.) When $r / M$ is large the value of $F_{t}$ approaches its flat spacetime counterpart. Recall that $\Omega=d \phi / d t$, where $t$ is the time of an observer at infinity. Because of the redshift effect at small values of $r$, orbits with the same value of $\Omega$ have very large proper tangential velocities at small $r / M$ : a fixed $\Omega$ corresponds to the ultrarelativistic limit when the orbit is close to the black hole. The second case is [Fig. 2(B)] geodesic motion, which satisfies Kepler's law $\Omega_{K}^{2} r^{3}=M$. The innermost (unstable) causal orbit is located at $r=$ $3 M$. Approaching $r=3 M$, the motion of the particle approaches the ultrarelativistic limit, which is manifested by the rapid growth of $F_{t}$. At larger radii the value of $F_{t}$ approaches the flat spacetime counterpart. This is detailed in Fig. 2(C).

Next, we study the radial, conservative component of the self-force. We start by checking the agreement of our numerical results with MSRP. MSRP predicts [7] that $a_{r}=0=c_{r}$ and that
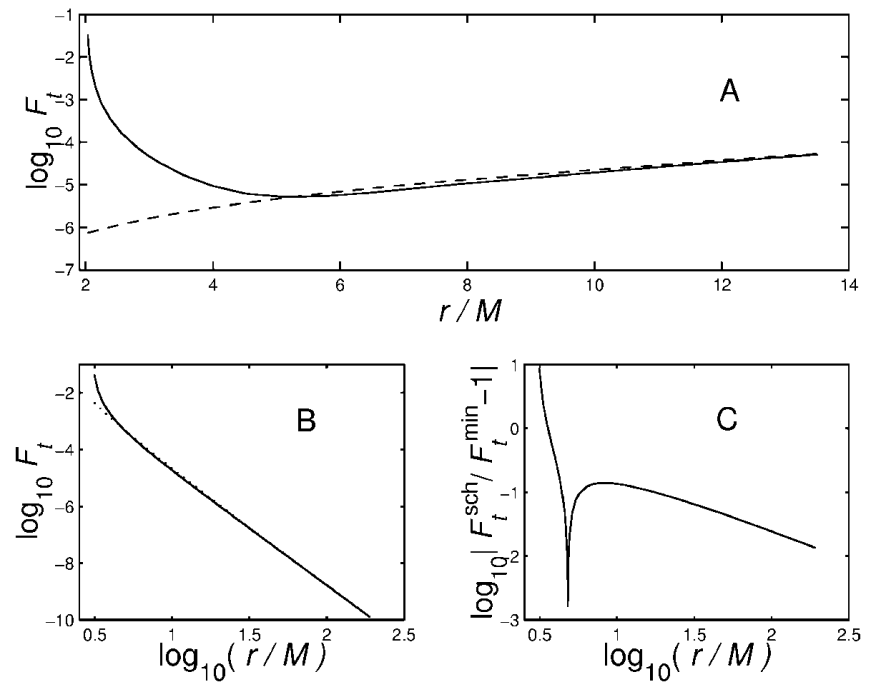

FIG. 2. The temporal component of the self-force $F_{t}$ as a function of $r / M$. Top panel (A): Fixed orbital velocity $\Omega=$ $3.2 \times 10^{-2} M^{-1}$ (nongeodesic orbits). $\Omega=\Omega_{K}$ when $r_{0}=$ 9.92M. Bottom panel (B): Keplerian orbits. Circular orbits in Schwarzschild are depicted by solid line, and in Minkowski by a dotted line. Bottom panel (C): The relative difference of the Schwarzschild and Minkowski results for Keplerian motion.

$$
\begin{aligned}
b_{r}=-\frac{q^{2}}{2 r^{2}} \frac{1}{\gamma \sqrt{-g_{t t}}}[ & 2{ }_{2} F_{1}\left(\frac{1}{2}, \frac{1}{2} ; 1 ; \frac{r^{2} \Omega^{2}}{1-2 M / r}\right) \\
& -\frac{1-3 M / r}{1-2 M / r} \\
& \left.\times{ }_{2} F_{1}\left(\frac{1}{2}, \frac{3}{2} ; 1 ; \frac{r^{2} \Omega^{2}}{1-2 M / r}\right)\right] .
\end{aligned}
$$

We check the accuracy of our numerical determination of the value of $b_{r}$ by comparison to Eq. (5). This check serves the twofold purpose of (i) checking the numerical code, and (ii) checking the compatibility of the analytical prediction of MSRP for the regularization function $h_{r}^{\ell}$ with the numerical determination of the bare force. Figure 3(A) shows the behavior of bare $F_{r}^{\ell}$, after summation over $m$ and $\omega$, as a function of $\ell$, and Fig. 3(B) displays ${ }^{\text {bare }} F_{r}^{\ell}-b_{r}$ as a function of $\ell$. This difference decreases like $\ell^{-2}$ for large values of $\ell$, which confirms the predictions of MSRP for the values of $a_{r}, b_{r}$, and $c_{r}$. (We emphasize that we cannot test numerically the prediction of MSRP that $d_{r}=0$.) Note that the radial ALD force vanishes, such that ${ }^{\text {ren }} F_{r}$ is the full self-force. Similar behavior is found also for Keplerian orbits at other values of $r_{0}^{*}$, and also for nongeodesic circular motion, with angular velocities both greater or smaller than $\Omega_{K}$.

The regularized component of the radial self-force ${ }^{\text {tail }} F_{r}^{\ell}$ is obtained by subtracting $b_{r} \equiv{ }^{\text {bare }} F_{r}^{\ell \rightarrow \infty}$ from each mode bare $F_{r}^{\ell}$. The total regularized force is then obtained by

$$
F_{r}^{\text {ren }}=\sum_{n=0}^{\ell}{ }^{\text {tail }} F_{r}^{n}+\mathcal{R}_{r}^{\ell+1}
$$



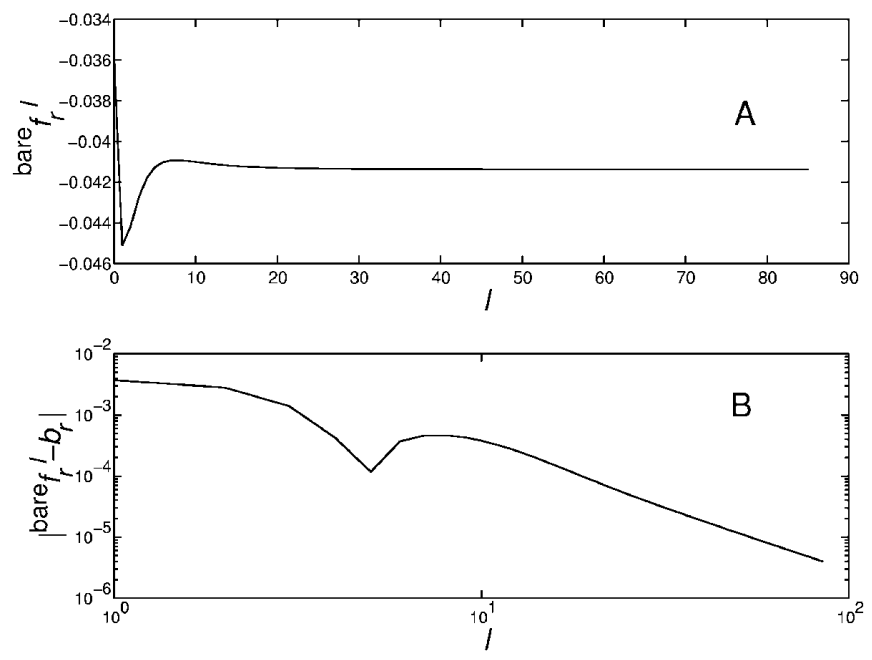

FIG. 3. The radial component of the $\ell$ multipole of the bare force as a function of $\ell$. For these data the orbit is at $r_{0}^{*}=3 M$. (A): ${ }^{\text {bare }} F_{r}^{\ell}$ for a geodesic orbit. (B) ${ }^{\text {bare }} F_{r}^{\ell}-b_{r}$ for the same orbit.

where the remainder $\mathcal{R}_{r}^{\ell+1}$ is given approximately by $\mathcal{R}_{r}^{\ell+1} \approx \ell^{2}\left({ }^{\text {bare }} F_{r}^{\ell}-b_{r}\right) \psi^{(1)}(\ell+1)$ for sufficiently large values of $\ell, \psi^{(1)}(x) \equiv d^{2} \ln \Gamma(x) / d x^{2}$ being the trigamma function. Note that for large arguments $\psi^{(1)}(x) \approx x^{-1}$. Figure 4 displays the regularized radial self-force for Keplerian [Fig. 4(A)] and non-Keplerian [Fig. 4(B)] orbits. The radial self-force in the far field limit $(r \gg M)$ is repulsive, and satisfies $F_{r}^{\text {ren }} \approx \alpha q^{2} M^{3} r^{-5}$, where $\alpha$ is a dimensionless parameter of order unity. A minimum- $\chi^{2}$ fit shows the exponent of $r$ to equal -5 and found $\alpha$ to equal unity, both with 3\% errors. However, in the strong field, the force law deviates from this simple relation, and grows faster. For the non-Keplerian orbits, we find that in the slow motion limit $\left(\Omega \ll \Omega_{K}\right)$ the radial force is proportional to $\Omega^{2}$. The exponent of $\Omega$ is found to be 2 with a $3 \%$ error. Combined with the result for Keplerian orbits, we find that for any circular orbit, in the far field and slow motion limits, the radial force is repulsive, and is given by

$$
F_{r}^{\mathrm{ren}} \approx \alpha q^{2}\left(G^{3} / c^{6}\right) M^{2} \Omega^{2} / r^{2} .
$$

This result explains the vanishing self-force in the static limit $[11,12]$. For faster motion the $\Omega^{2}$ law no longer hold any more. In fact, for $\Omega>\Omega_{K}$ we find that the radial selfforce varies rapidly, and eventually changes from repulsive to attractive. The radial self-force does not cause a net change in the energy of the particle. However, if the orbit has a nonzero eccentricity, this force induces an additional precession of the periastron, which in the slow motion or far field limits is retrograde. This precession has an effect on the frequencies of the emitted radiation.

Our results show that the self-force can be calculated for a simple, although nontrivial, problem. MSRP was found to be useful also for other cases, e.g., static scalar and electric charges in Schwarzschild [11], scalar and electric charges in circular motion in flat spacetime [13], and gen-
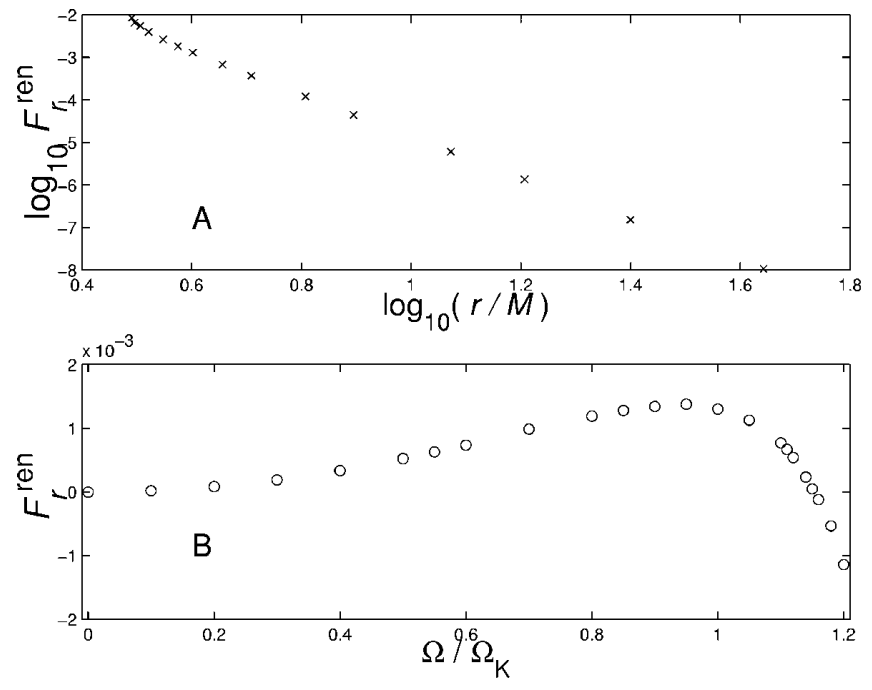

FIG. 4. The regularized radial self-force. Top panel (A): $F_{r}^{\text {ren }}$ as a function of $r / M$ for Keplerian (geodesic) orbits. Bottom panel (B): $F_{r}^{\text {ren }}$ as a function of $\Omega / \Omega_{K}$ for $r_{0}^{*}=4 M$.

eral radial motion of scalar charges in spherical symmetry [14]. A closely related approach was used also for a mass point in radial free fall in Schwarzschild [15]. We hope that similar methods can be used for more realistic cases, which may be relevant for the orbital evolution of compact objects around black holes.

I thank Scott Hughes and Kip Thorne for valuable discussions. I am indebted to Amos Ori for many stimulating discussions and for letting me use his results before their publication. This research was supported by NSF Grants No. AST-9731698 and No. PHY-9900776, and by NASA Grant No. NAG5-6840.

[1] É.É. Flanagan and S. A. Hughes, Phys. Rev. D 57, 4566 (1998).

[2] S. A. Hughes, Phys. Rev. D 61, 084004 (2000).

[3] T. C. Quinn and R. M. Wald, Phys. Rev. D 56, 3381 (1997).

[4] Y. Mino, M. Sasaki, and T. Tanaka, Phys. Rev. D 55, 3457 (1997).

[5] T. C. Quinn and A. G. Wiseman (to be published).

[6] A. Ori, Phys. Lett. A 202, 347 (1995); Phys. Rev. D 55, 3444 (1997).

[7] A. Ori (to be published).

[8] L. Barack and A. Ori, Phys. Rev. D 61, 061502(R) (2000).

[9] A. G. Wiseman (to be published).

[10] W. G. Anderson and É. É. Flanagan (to be published).

[11] L. M. Burko, Classical Quantum Gravity 17, 227 (2000).

[12] A. I. Zel'nikov and V.P. Frolov, Sov. Phys. JETP 55, 191 (1982); A. G. Wiseman, Phys. Rev. D 61, 084014 (2000); A. E. Mayo, Phys. Rev. D. 60, 104044 (1999).

[13] L. M. Burko, Am. J. Phys. 68, 456 (2000); L. M. Burko (unpublished).

[14] L. Barack and A. Ori (to be published); L. Barack and L. M. Burko (to be published).

[15] C. O. Lousto, Phys. Rev. Lett. (to be published). 